\title{
Aggressive Periodontitis in a Nigerian Teaching Hospital
}

\author{
${ }^{1}$ Solomon Olusegun Nwhator, ${ }^{2}$ lyobosa Uhunmwangho, ${ }^{3}$ Benedict Chukwuma, ${ }^{4}$ Osagie Ikponmwosa
}

\section{ABSTRACT}

Aim: These case series were aimed at highlighting late presentations of aggressive periodontitis (AP) in a teaching hospital as well as proffering possible reasons for such presentations which would serve as part of the solution to prevent such presentations in the future.

Background: Aggressive periodontitis is a severe form of destructive periodontitis traditionally believed to present around puberty. However, many cases seen in a teaching hospital presented much later for yet-to-be explained reasons.

Case description: Seven patients referred to the specialist periodontal clinic of a Nigerian teaching hospital presented with clinical features consistent with AP. Most of the patients were over twenty and some over thirty years of age.

Conclusion: Aggressive periodontitis patients seen in our center were often outside the traditional age brackets. The range of treatment options available to the patients were under-utilized due to serious financial constraints.

Clinical significance: Aggressive periodontitis comes with serious psychological challenges and severe morbidity. Prompt diagnosis and effective management hold the key to success It is important to investigate why many of the cases seen in our center presented that late. Could be due to ignorance and poverty or could be due to failure of dentists recognize these cases and consequent misdiagnosis? Further studies are needed to answer these questions.

Keywords: Aggressive periodontitis, Late presentation, Poverty, Ignorance, Misdiagnosis.

How to cite this article: Nwhator SO, Uhunmwangho I, Chukwuma B, Ikponmwosa O. Aggressive Periodontitis in a Nigerian Teaching Hospital. J Contemp Dent Pract 2014;15(4): 518-522.

\section{Source of support: Nil}

Conflict of interest: None declared

\footnotetext{
${ }^{1}$ Senior Consultant, ${ }^{2}$ Senior Registrar, ${ }^{3,4}$ Registrar

1,2Department of Preventive Dentistry, University of Abuja Teaching Hospital, Abuja, Nigeria

${ }^{3}$ Department of Oral and Maxillofacial Surgery, University of Abuja Teaching Hospital, Abuja, Nigeria

${ }^{4}$ Department of General Dental Practice, University of Abuja Teaching Hospital, Abuja, Nigeria
}

Corresponding Author: Solomon Olusegun Nwhator Senior Consultant, Department of Preventive Dentistry University of Abuja Teaching Hospital, Abuja, Nigeria, Phone: 2348093690829, e-mail: periodontologist2010@gmail.com

\section{INTRODUCTION}

Aggressive periodontitis (AP) is a severe rapidly progressing rare variant of chronic periodontitis ${ }^{1}$ causing clinical attachment loss in an otherwise healthy individual and the amount of tissue loss is inconsistent with the amount of microbial deposits; and with a familial aggregation of diseased individuals. ${ }^{2}$ The condition primarily - but not exclusively - affects younger patients. ${ }^{3}$ Thus, the terms 'juvenile periodontitis' and 'early-onset periodontitis' have been replaced by the so called $\mathrm{AP}-\mathrm{A}$ better reflection of its clinical course.

Direct and indirect bacterial effects influencing the host immune response play a significant part in the etiology of AP comparable to chronic periodontitis. ${ }^{4}$ Though the progression of attachment and bone loss may be self-limiting 5 for which several reasons have been propounded, the disease is associated with considerable morbidity 6 in patients who abscond from treatment regimen or who for any reasons, have not received treatment.

The goals of periodontal therapy in AP are to alter or eliminate the microbial etiology - primarily Aggregatibacter actinomycetemcomitans ${ }^{7}$ - and contributing risk factors for periodontitis; thereby arresting progression of disease and preserving the dentition in comfort, function and appropriate esthetics; and to prevent recurrence of the disease. Early detection is key to obtaining good treatment outcome. ${ }^{8}$

The average age of onset of AP is between puberty and 20 years, however patients present before 30 years. In a 'profile of chronic periodontitis and AP among Senegalese', the average age of presentation was put at $28.1 \pm 8.9$ and had lost $<3$ teeth and the average number of missing teeth was $1.29 /$ subject. $^{9}$

Even though there have been attempts to analyze AP biochemically and microbiologically, ${ }^{10.11}$ there is no specific way to discover AP early. Currently, early detection depends primarily on clinical and radiographic examination and exclusion of other systemic conditions that can cause tooth loss.

In our center situated in the geographical center of Nigeria the most populous nation in West Africa, the age of presentation may commonly be $>30$ years. In the experience of the authors, such late presentation comes with extreme morbidity and almost hopelessness- hopeless dental situation attributable to years of unattended periodontal destruction. 

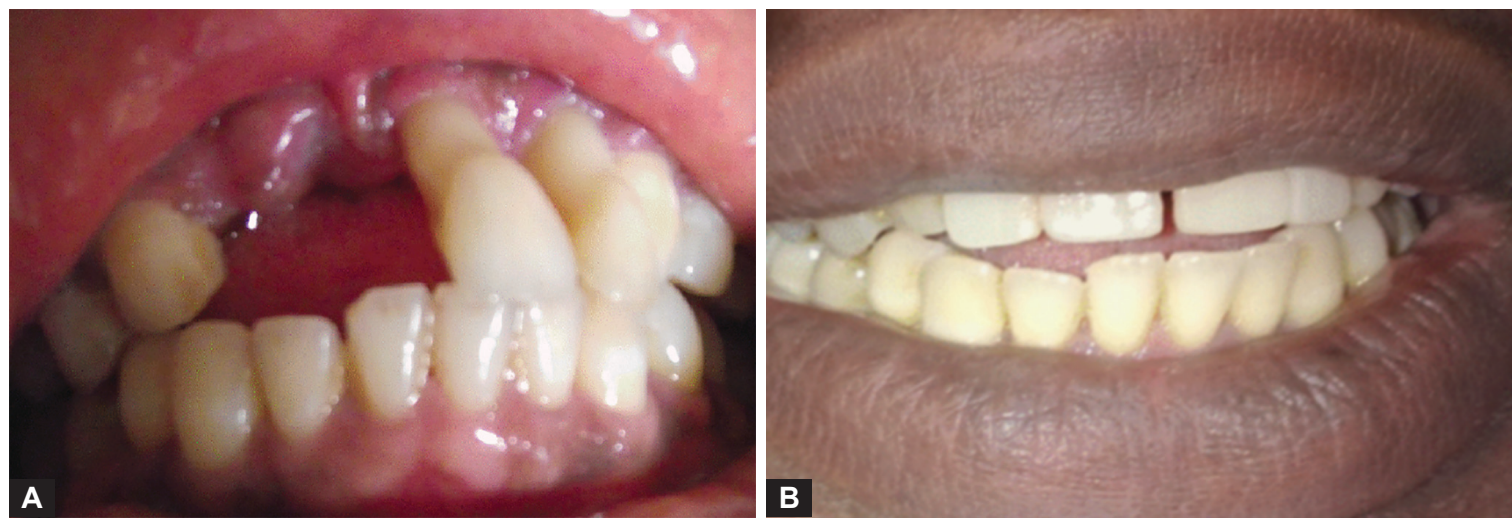

Figs 1A and B: 37 years female shows lost teeth 11 and 12, before and after treatment (case 1)

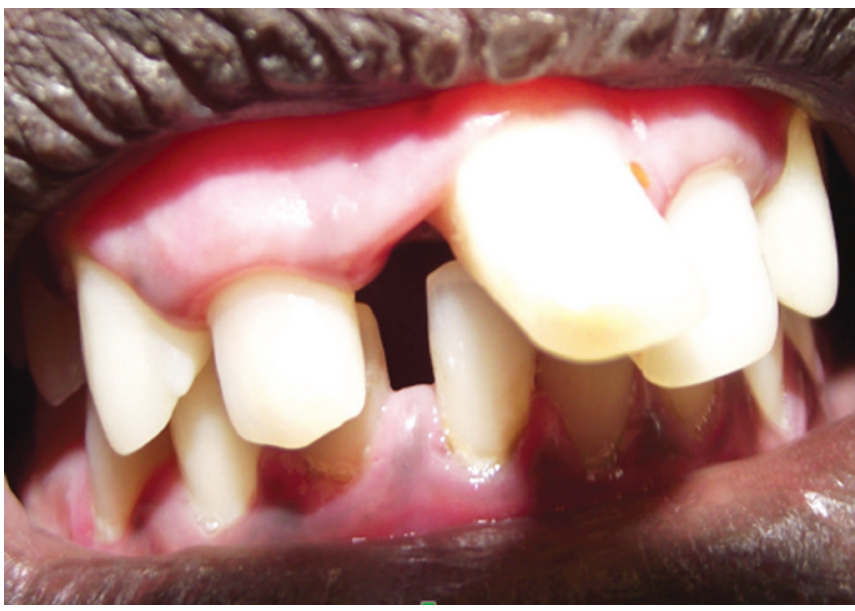

Fig. 2: 14 years male showing drifted tooth 11 and good oral hygiene (case 2)

The authors were baffled by the consistent lateness in the presentation of cases seen over 6 months within at the hospitals specialist periodontal clinic. The present report is aimed at highlighting this disturbing situation with a view to discovering the reasons for it and possibly proffering solutions.

\section{CASE REPORTS}

\section{Case 1: 37 Years Female}

A 37-year-old female patient presented at the specialist periodontal clinic on referral from the oral diagnosis clinic of the hospital with the complain of 'missing teeth' request for 'replacement' teeth (Fig. 1). On examination, the patient was an otherwise healthy lady with no evidence of systemic diseases. Intraoral examination revealed missing teeth 11 and 12. Tooth 21 and 22 showed marked bone loss with most of their roots exposed.

A provisional diagnosis of AP was made and periapical radiographs revealed angular and crescentic bone loss around teeth $26,31,41$ and 46 . Since, the periodontal destruction and radiographic evidence of bone loss were limited to central incisors and first permanent molar, a definitive diagnosis of localized AP was made.
The nature of the disease was well-explained to the patient. She was placed on a course of oral $250 \mathrm{mg} 6$-hourly tetracycline for 2 weeks. There was a serious financial restrain hence the patient offered extraction of teeth 21 and 22 and replacement of all maxillary incisors and scaling and root planning (SRP). Teeth were replaced with acrylic dentures due to financial constraints.

\section{Case 2: 14 Years Male}

A 14-year-old male on referral from the oral diagnosis clinic presented with missing tooth 12 and severe drifting of tooth 11 (Fig. 2). The oral hygiene was 'very' good and there was no history or evidence of any systemic illness. Periapical radiographs revealed angular bone loss around 26 and vertical bone loss around tooth 41 . A diagnosis of localized AP was made.

After explaining the nature of the disease to the patient and the mother, a course of $100 \mathrm{mg}$ doxycycline capsules, 12 hourly for 2 weeks was prescribed. The patient's mother appeared only interested in dentures and patient never attended follow-up clinics hence further treatment could not be given.

\section{Case 3: 25 Years Female}

A 25-year-old lady was seen at the specialist periodontal clinic on referral from the oral diagnosis clinic of the dental hospital (Fig. 3). Her only reason for attendance was to obtain 'replacement' teeth as many of her teeth were missing.

History and examination revealed an otherwise healthy patient with ten missing teeth namely-11, 12, 13, 14, 21, $22,23,24,31$ and 33 . Her oral hygiene was fair and there were no painful teeth. The 41 distolingually rotated and periapical radiograph of the 36 revealed extensive vertical and periradicular bone loss. Because 3 other permanent teeth were involved apart from the central incisors and the first permanent molars, a diagnosis of generalized AP was made. However, no systemic illnesses were found. After a course of $100 \mathrm{mg}$ doxycycline capsules, 12 hourly for 2 weeks, SRP as well as partial dentures replacing all missing teeth. 


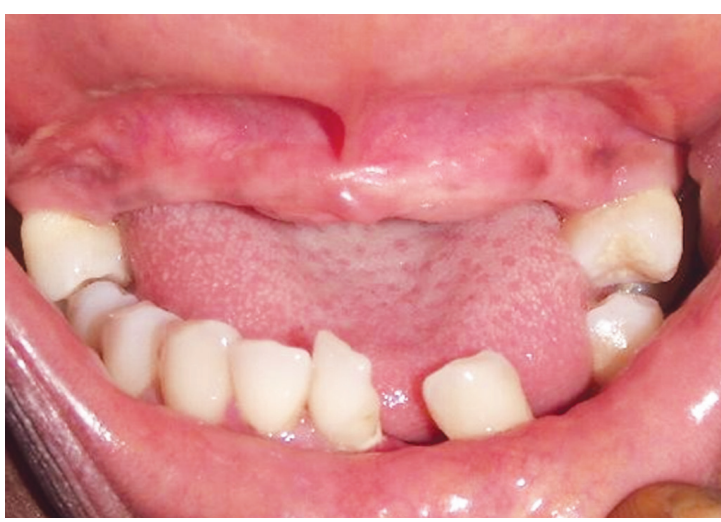

Fig. 3: 25 years female showing missing maxillary anterior sextant (case 3)

\section{Case 4: 19 Years Female}

A 19-year-old female patient presented at the specialist periodontal clinic on referral from the oral diagnosis clinic with a history of missing mobile teeth (Fig. 4). The reason fro attendance was for extraction. History and examination revealed an otherwise healthy teenage girl good oral hygiene but mobile and drifting teeth. The 16 was missing while 11, 21 and 26 were grade 3 mobile. Periapical radiographs revealed vertical bone loss related to the maxillary central incisors and left mandibular molars.

The patient had been a previous dose of systemic tetracyclines but had absconded from treatment. After another round of explanation of the nature of the disease

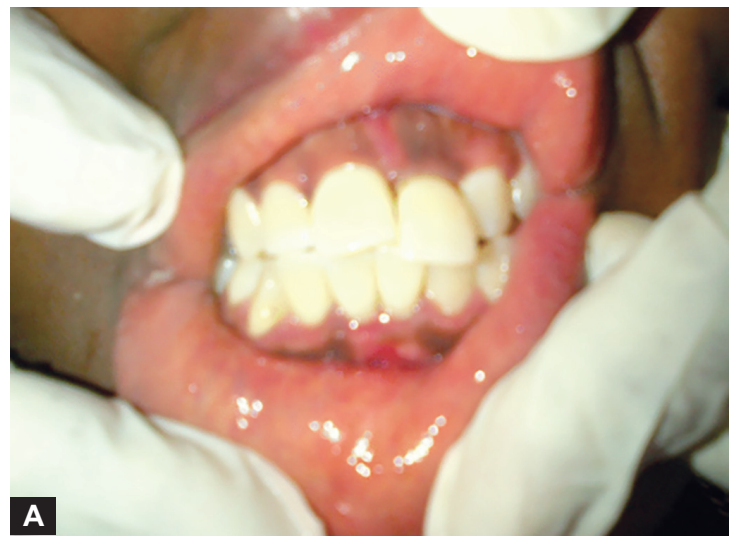

Figs $4 A$ and B: 19 years female showing drifted tooth 11 and missing tooth 26 (case 4)

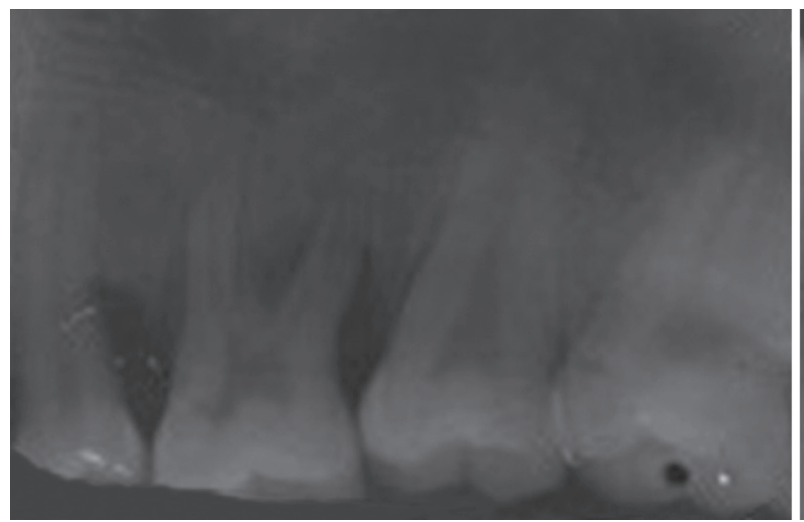

Fig. 5: 21 years female showing angular bone loss around teeth 34, 35, 36, 45 and 46 (case 5)

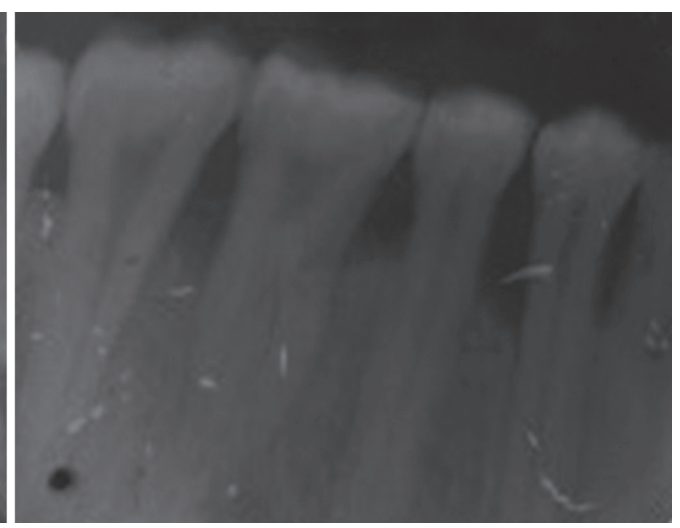

and counseling to keep appointments, a repeat those of tetracycline was given.

Grade 3 mobile 26 was subsequently extracted and patient was offered SRP for other involved teeth. Patient however did not keep her appointments and only showed up about 5 months later requesting for another extraction of a now 3rd degree mobile lower molar. The advise to get the maxillary centrals extracted with immediate denture fitted was not heeded again due to financial restraints.

\section{Case 5: 21 Years Female}

A 21-year-old otherwise healthy lady presented at the specialist periodontal clinic of the hospital with a complain missing teeth and request for 'replacement' teeth (Fig. 5). Teeth 31 and 41 were missing. Periapical radiographs revealed angular bone loss related to teeth $34,35,36,45$ and 46. Offer of SRP for involved teeth was not well received due to financial constraints but systemic antibiotics were prescribed. Teeth 31 and 41 were replaced with acrylic dentures due to financial constraints. Attempts to arrange follow-up visits were rebuffed by the patient.

\section{Case 6: 20 Years Female}

An adult female in her 40 seconds was seen at the specialist periodontal clinic on referral from the oral diagnosis clinic (Fig. 6). She complained of mobile teeth and on examination,

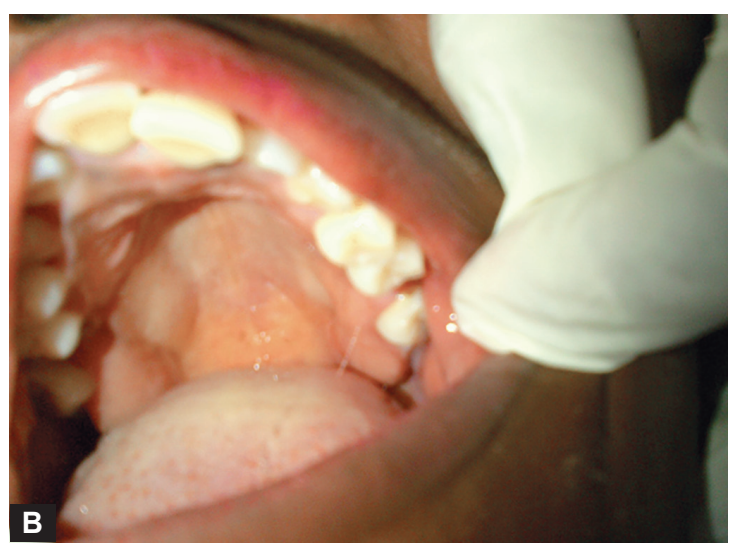

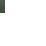



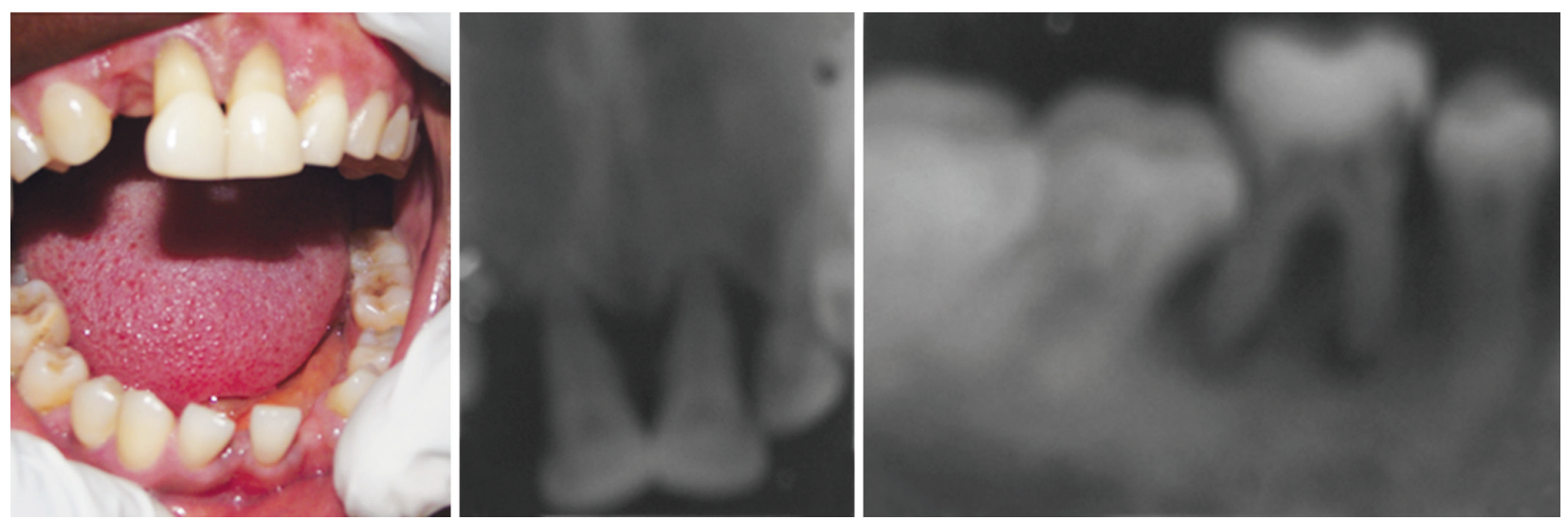

Fig. 6: Female/adult showing severe periodontal destruction 11 and 21, missing 12 and 32 and gingival recession around 22 (case 6)

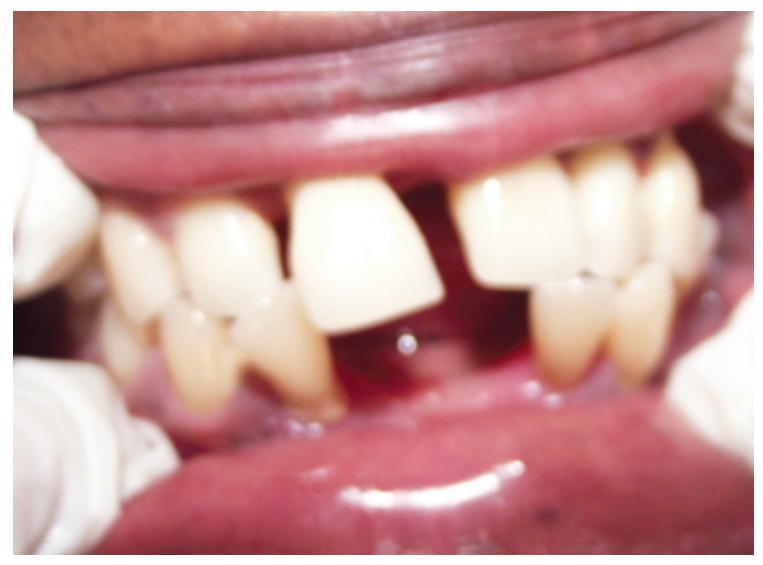

Fig. 7: 20 years female showing missing teeth 31 and 41 (case 7)

gross periodontal destruction was noticed around teeth 11 and 21 with grade III mobility and Miller's class IV gingival recession. Tooth 12 and 32 were missing while tooth 22 presented with Miller's class II gingival recession. There was radiological evidence of severe periodontal bones loss around tooth 46. A diagnosis of extremely-late presenting generalized AP was made on clinical and radiological ground.

\section{Case 7: 20 Years Female}

A 20-year-old lady was seen at the specialist periodontal clinic on referral from the oral diagnosis clinic of the dental hospital (Fig. 7). Her complains were missing and mobile teeth for which she needed 'replacement' teeth. History and examination of this otherwise healthy lady revealed no systemic health problems but intraoral examination revealed anterior maxillary drifting and spacing as well as missing 31 and 41. Periapical radiographs revealed angular bone loss around 16, 26, 36 and 46. A diagnosis of localized AP was made, systemic doxycycline, prescribed while SRP and prosthesis were offered.

\section{Cases 8 and 9: 31 and 33 Year Females}

Cases 8 and 9 were 31 and 33-year old ladies presenting with a similar history of missing lower incisors and mobile teeth.
Both are considered together for space constraints and their similarity of presentation. One was already an acrylic denture wearer since teenage years who was requesting attention because she wanted to avoid further extractions while the second patient was a nurse. The diagnosis of localized AP was made in each instance, systemic doxycycline prescribed, SRP offered and hopeless teeth planned for extraction. Prosthetic rehabilitation was planned in both instances using acrylic partial dentures for financial reasons.

\section{DISCUSSION}

Aggressive periodontitis is considered rare but it is more prevalent in Africans than among Caucasians. It is generally considered an early-presenting disease with a few exceptional cases seen after puberty. There is insufficient data to conclude that Nigerian patients start to experience AP any earlier than reported in literature but late presentation appears to be norm from our experience as a handful of publications about the Nigerian experience have been published but none has given enough detail to the particularly late presentation of Nigerian patients suffering from AP.

Contrary to the overwhelming evidence in literature suggesting an only onset and presentation described with terms like 'early age,, ${ }^{12}$ 'younger age, ${ }^{2}$ most of the patients referred to the specialist periodontal clinic in our hospital were above 30 years of age. Again, it needs be emphasized that the concern of the authors of the present paper was age of presentation rather than age of onset.

In the opinion of the authors, the fact that five of the nine patients seen were over 30 years of age with one being 37 years of age was quite significant.

It was almost impossible to correctly ascertain the age of the onset of the patients referred to our clinic since most were either first timers or infrequent dental clinic attendees. From the inability of the patients to pay for qualitative tooth replacement options, it appears that the two main factors responsible for our observations are ignorance and poverty. 
Morbidity judged by the number of lost teeth was quite significant in our study. The loss of teeth in the esthetic zone without seeking immediate care sparks of extreme ignorance, extreme poverty or both. This is evident in cases 1,3 and 7 . The same inferences can be made with the obvious malocclusion of cases 2 and 4 .

Considering the fact that 7 of the 9 cases seen were single females points attention to another neglected fact-social implications of AP. Were these patients seeking treatment because they were thinking of serious relationships or marriage? Was their condition affecting their chances of attracting a suitable spouse?

The fact that these were all females who ordinarily would be more conscious of their appearance makes the situation even more worrisome. Virtually all subjects presented at the clinic for the purpose of seeking prosthesis to replace missing teeth buttresses the opinion of extreme poverty and ignorance which are poorly reported in literature.

\section{CONCLUSION AND RECOMMENDATIONS}

Most cases of AP seen in our center presented later than usual with severe periodontal destruction. Poverty and ignorance seems to affect the age of presentation as well as morbidity of AP at least in our center.

Massive public awareness drives as well as possible sponsorship of poor affected individuals would go a long way toward rehabilitating affected individuals.

\section{CLINICAL SIGNIFICANCE}

Aggressive periodontitis comes with serious psychological challenges and severe morbidity. Prompt diagnosis and effective management hold the key to success. It is important to investigate why many of the cases seen in our center presented that late. Could be due to ignorance and poverty or could be due to failure of dentists recognize these cases and consequent misdiagnosis? Further studies are needed to answer these questions.

\section{REFERENCES}

1. Roshna T, Nandakumar K, 'Generalized aggressive periodontitis and its treatment options: case reports and review of the literature,' Case Reports in Medicine, vol. 2012, Article ID 535321, 17 pages, 2012. doi:10.1155/2012/535321.

2. Llorente MA, Griffiths GS. Periodontal status among relatives of aggressive periodontitis patients and reliability of family history report. J Clin Periodontol 2006 Feb;33(2):121-125. PubMed PMID: 16441736.

3. Masamatti SS, Kumar A, Virdi MS. Periodontal diseases in children and adolescents: a clinician's perspective part. Dent Update. 2012 Oct;39(8):541-544, 547-548, 551-552.

4. Shaddox LM, Huang H, Lin T, Hou W, Harrison PL, Aukhil I, Walker CB, Klepac-Ceraj V, Paster BJ. J Dent Res. 2012 Oct; 91(10):927-933.

5. Stein JM, Machulla HK, Smeets R, Lampert F, Reichert S. Human leukocyte antigen polymorphism in chronic and aggressive periodontitis among Caucasians: a meta-analysis. J Clin Periodontol 2008 Mar;35(3):183-192.

6. Cho CM, You HK, Jeong SN. The clinical assessment of aggressive periodontitis patients. J Periodontal Implant Sci 2011 Jun;41(3):143-148.

7. Johansson A. Aggregatibacter actinomycetemcomitans leukotoxin: a powerful tool with capacity to cause imbalance in the host inflammatory response. Toxins (Basel). 2011 Mar;3(3): 242-259.

8. Levin L. Aggressive periodontitis: the silent tooth killer. Alpha Omegan 2011 Fall-Winter; 104(3-4):74-78.

9. Benoist HM, Seck-Diallo A, Diouf A, Yabbre S, Sembene M, Diallo PD. Profile of chronic and aggressive periodontitis among Senegalese. J Periodontal Implant Sci. 2011 Dec;41(6): 279-284.

10. Elerson Gaetti J Jr, Maria Dias BJ, Marquezim LA, et al. Occurrence of Actinobacillus actinomycetemcomitans in patients with chronic periodontitis, aggressive periodontitis, healthy subjects and children with gingivitis in two cities of the state of São Paulo, Brazil. J Appl Oral Sci. [serial on the Internet]. 2006 June [cited $2013 \mathrm{Feb} 25$ ];14(3):153-156.

11. Haigh BJ, Stewart KW, Whelan JRK, Barnett MPG, Smolenski GA, Wheeler TT. Alterations in the salivary proteome associated with periodontitis. J Clin Periodontol 2010;37:241-247.

12. Salman A, Meethil A. An unusual presentation of generalized aggressive periodontitis with multiple impacted supernumerary teeth. Eur J Dent 2012 Jul;6(3):335-339. 